\title{
Simple feedback of colonoscopy performance improved the number of adenomas per colonoscopy and serrated polyp detection rate
}

\section{(ㄷ)(1) $(2) \Theta$}

Authors

Osamu Toyoshima ${ }^{1,2}$, Shuntaro Yoshida ${ }^{1,2}$, Toshihiro Nishizawa ${ }^{1,3}$, Tadahiro Yamakawa ${ }^{1}$, Toru Arano ${ }^{1,4}$, Yoshihiro Isomura ${ }^{1,5}$, Takamitsu Kanazawa' ${ }^{1,6}$, Hidehiko Ando7, Yosuke Tsuji², Kazuhiko Koike²

Institutions

1 Gastroenterology, Toyoshima Endoscopy Clinic, Tokyo, Japan

2 Department of Gastroenterology, Graduate School of Medicine, The University of Tokyo, Tokyo, Japan

3 Department of Gastroenterology and Hepatology, International University of Health and Welfare, Chiba, Japan

4 Department of Gastroenterology, The Fraternity Memorial Hospital, Tokyo, Japan

5 Department of Gastroenterology, Kyoundo Hospital, Tokyo, Japan

6 Department of Gastrointestinal Surgery, JR Tokyo General Hospital, Tokyo, Japan

7 Gastroenterology, Ando Family Clinic, Tokyo, Japan

submitted 8.6.2020

accepted after revision 26.10 .2020

Bibliography

Endosc Int Open 2021; 09: E1032-E1038

DOI 10.1055/a-1393-5469

ISSN 2364-3722

(C) 2021. The Author(s).

This is an open access article published by Thieme under the terms of the Creative Commons Attribution-NonDerivative-NonCommercial License, permitting copying and reproduction so long as the original work is given appropriate credit. Contents may not be used for commercial purposes, or adapted, remixed, transformed or built upon. (https://creativecommons.org/licenses/by-nc-nd/4.0/)

Georg Thieme Verlag KG, Rüdigerstraße 14,

70469 Stuttgart, Germany

Corresponding author

Toshihiro Nishizawa, MD, PhD, Professor, Department of

Gastroenterology and Hepatology, International University of Health and Welfare, Narita Hospital, 852 Hatakeda, Narita, Chiba 286-8520, Japan

Fax: +81-476-35-5586

nisizawa@kf7.so-net.ne.jp

\section{ABSTRACT}

Background and study aims High-quality endoscopy requires improvement of not only the adenoma detection rate (ADR) but also the serrated polyp (SP) detection rate and the mean number of adenomas per positive procedure $(\mathrm{MAP}+)$. We evaluated whether a simple feedback of colonoscopy performance improves those quality indicators using propensity-score matching.

Patients and methods Eleven endoscopists were evaluated regarding colonoscopy performance including ADRs, SP detection rates, mean numbers of adenomas per procedure (MAPs), and MAPs + with their ranking in the clinic. Endoscopic performance was compared before and after the feedback.

Results Colonoscopies were performed for 874 patients before the feedback and 1,272 patients after the feedback. Using propensity-score matching, 803 patients before the feedback and 803 patients after the feedback were matched. ADR after the feedback was significantly higher than that before the feedback ( $50.8 \%$ and $40.8 \%$, respectively). MAP after feedback was significantly larger than that before the feedback ( 0.92 and 0.69 , respectively), as well as $\mathrm{MAP}+(1.96$ and 1.69 , respectively). Clinically significant SP detection rate was also improved from $10.0 \%$ to $14.9 \%$. Conclusions Feedback including ADR, MAP, MAP+, and clinically significant SR detection rate could improve on those quality indicators. Further studies are needed to effectively prevent colorectal cancer in colonoscopy practice.

\section{Introduction}

Colorectal cancer (CRC) is the major cause of cancer-related mortality worldwide. Removal of premalignant polyps dramatically prevents CRC. High-quality colonoscopy plays an essential role in both the early detection and prevention of CRC through the detection and removal of colorectal adenomas [1]. Up to $30 \%$ of interval CRCs were estimated to be caused by incomplete polyp resection [2]. Kaminski et al. demonstrated that an adenoma detection rate (ADR) of $<20 \%$ among colonosco- 
pists was associated with a higher rate of interval CRC [3]. Corley et al. proved that every $1 \%$ increase in ADR was associated with a $3 \%$ decrease in interval CRC incidence [4]. Furthermore, an increased $A D R$ was associated with a reduced risk of interval CRC and death. These studies provide strong evidence that colonoscopists should aim for higher ADRs.

Interval CRCs disproportionally often arise in the proximal colon. This disproportion may be due to both biological characteristics and missed or incomplete resection of serrated polyps (SPs), which are considered as precursors in $20 \%$ to $30 \%$ of CRC cases [5-7]. Therefore, missing SPs are believed to be an essential factor in interval CRC development, and improvement of SP detection has a great impact on interval CRC incidence $[6,8,9]$.

Endoscopy training improved the ADR [10-13], mean number of adenomas per procedure (MAP) [14], sessile serrated lesion (SSL) detection rate [15], and proximal SP detection rate [16]. Lam et al. reported that colonoscopy training improved interval CRC rates from $0.15 \%$ to $0.08 \%$ and early interval CRC rates from $0.07 \%$ to $0.04 \%$ [17].

Meanwhile, several studies observed that implementation of the ADR, MAP, and mean number of adenomas per positive procedure (MAP+) was also a crucial indicator of colonoscopy quality $[18,19]$ because a focus on the ADR may lead to an "one and done" phenomenon [14, 20].

Although a simple feedback of colonoscopy performance has been reported to improve ADR [11], it is still unclear if the feedback improves the SP detection rate and MAP+. Furthermore, the assessment for ADR improvement needs to minimize selection bias and potential confounding factors. However, there is no report using propensity-score matching to assess ADR improvement. Therefore, we evaluated whether feedback of colonoscopy performance improves not only the ADR but also the SP detection rate and MAP+ using propensity-score matching.

\section{Methods}

\section{Study design}

This study used a retrospective longitudinal cohort design and propensity-score matching for baseline patients' characteristics.

We enrolled patients who underwent colonoscopy at Toyoshima Endoscopy Clinic from May to August 2018. The approximate colonoscopies per annum at the endoscopy-specialized clinic was 4,000. In December 2018, the endoscopists who conducted colonoscopy during the period simply had their following colonoscopy performance fed back: their accurate numerical ADRs, SP detection rates, MAPs, and MAPs + including patients' average age, sex ratio, colonosope insertion time, and withdrawal time. Doctor-specific ADRs were presented in a bar chart and ranked at a meeting on December 2018. The SP detection rate, MAP, and MAP + were also presented in the same way. The director of the clinic commended the top three endoscopists at the meeting and suggested the target ADR of $\geq 40 \%$ for the purpose of effectively preventing CRC. The director informed the endoscopists that their colonoscopy performance would be measured again next year. In addition to the sin- gle meeting, individual interviews between the director and each endoscopist were also held. In the interviews, the director disclosed the individual score for each endoscopist. Moreover, we collected data from colonoscopies performed by the same endoscopists from April to August 2019. We compared colonoscopy performance before and after the feedback.

This retrospective study was approved by the Ethical Review Committee of the Hattori Clinic on September 6, 2019 (approval no.S1909-U06). Written informed consent was obtained from the participants. All clinical investigations were conducted according to the ethical guidelines of the Declaration of Helsinki.

\section{Patients}

Colonoscopy was performed to evaluate symptoms (i. e., hematochezia, rectal bleeding, abdominal symptoms, abnormal bowel habits, and anemia), for screening including positive fecal immunochemical test, or for colorectal polyp surveillance. This study included patients who had an endoscopy indicated for gastrointestinal symptoms or positive fecal immunochemical test results, and for colorectal polyp surveillance. We excluded patients who were not willing to undergo removal of colorectal polyps, patients who had indications of surveillance for CRC or inflammatory bowel disease, patients who had undergone colorectal surgery except appendectomy, and patients who had indications for treatment such as colorectal polypectomy and hemostasis. We excluded colonoscopy procedures that did not reach the cecum due to colonic stenosis, procedures with poor bowel preparation disabling complete observation, and procedures that did not remove all the polyps due to consumption of an antithrombotic drug, the large size and/or number of polyps, or difficulty of polypectomy [21]. We removed the polyps that are less than 10 , those with a diameter of $\leq 15 \mathrm{~mm}$, and those with a cumulative diameter $\leq 30 \mathrm{~mm}$, at a single colonoscopy procedure [22].

\section{Endoscopists}

The endoscopists did not know their own exact numerical ADR before the feedback in December 2018. Thirteen endoscopists performed colonoscopies during the considered period before the feedback. Of them, 11 endoscopists conducted colonoscopy also after the feedback and were enrolled in this study. The lifetime colonoscopy experiences of the 11 endoscopists at the time of enrollment were 20,000,11,000,10,000, 10,000, $7,000,5,000,5,000,5,000,5,000,4,000$, and 2,000, respectively.

\section{Diagnosis of polyps}

Lesions diagnosed as adenomas or clinically significant SPs (CSSPs) were removed. A CSSP was defined as any SSL, any traditional serrated adenoma, hyperplastic polyp $\geq 1 \mathrm{~cm}$ anywhere in the colon, or hyperplastic polyp $\geq 5 \mathrm{~mm}$ and proximal to the sigmoid colon $[15,23,24]$. We chose CSSP instead of SSL as SP definition because a short surveillance interval is recommended for CSSPs as well as SSLs, and all CSSPs were removed in clinical practice [25]. We primarily diagnosed a polyp as a SSL according to the following findings: indistinctive border, mucus 
cap, normal or pale color, none or dilated vessels, and/or dilated crypts [26]. Polyp size was measured by placing a closed snare or forceps, which has a thickness of $2 \mathrm{~mm}$, against the lesion. Lesions were removed either by hot or cold polypectomy using a snare or forceps or by endoscopic mucosal resection on examination day.

All resected specimens were examined histologically under hematoxylin and eosin staining. One experienced gastrointestinal pathologist diagnosed the polyps according to the World Health Organization criteria [27]. An advanced adenoma was defined as an adenoma with a villous component, with a size $>10 \mathrm{~mm}$, or with high-grade dysplasia based on the World Health Organization definition $[27,28]$. Low-risk adenomas were defined as one or two tubular adenomas less than $10 \mathrm{~mm}$, and high-risk adenomas were defined as at least one advanced adenoma or three or more adenomas [29]. Only lesions that were histologically confirmed as adenomas or CSSPs were counted [30].

\section{Colonoscopy}

Small shaking, jiggling, and right-turn-shortening maneuvers have been frequently used for colonoscope insertion. Extra gas and liquid were aspirated and removed as much as possible. $\mathrm{CO}_{2}$ was administered for colonic insufflation. Colonoscopies were performed under conscious sedation with midazolam and/or pethidine hydrochloride [31,32]. In the absence of contraindications, we administered 10 to $20 \mathrm{mg}$ of scopolamine butylbromide.

The observation time for withdrawal of the colonoscope was standardized as at least $6 \mathrm{~min}$. The colonoscope was sequentially withdrawn as the prescribed position changed: the ascending colon was examined in the left lateral decubitus position, the transverse colon was examined in the supine position, the descending colon was examined in the right lateral position, and the sigmoid colon and rectum were examined in the left lateral position [12, 21, 22, 33, 34].

Patients involved in this study underwent colonic preparation with $2 \mathrm{~L}$ of polyethylene glycol solution administered 4 to 5 hours before the procedure. Magnesium citrate or polyethylene glycol solution was added when the stool was not clear liquid. The quality of bowel preparation was graded as A (all colon segments empty and clean or minor amount of fluid in the gut that was easily removed by suction), B (at least one colon segment with residual amounts of brown liquid or semisolid stool that could be easily removed or displaced), C (at least one colon segment with only partially removable stool preventing complete visualization of mucosa), or D (at least one colon segment that could not be examined due to the presence of remaining solid stool). The following colon segments were estimated: cecum/ascending colon, transverse colon, descending colon, sigmoid colon, and rectum [25]. Patients graded as D during the colonoscopy were excluded from the study.

Patients underwent colonoscopy with an Elite CF290 endoscopy system (CV-290 and CLV-290, Olympus, Japan) with a 290 series colonoscope (CF-HQ290Z, CF-HQ290, or PCF-H290Z, Olympus, Japan) or a 260 series colonoscope (PCF-PQ260 or CF-H260). Narrowband imaging was available for all scopes.
PCF-H290Z and PCF-PQ260 were used in patients aged 80 years or older, patients aged $\geq 70$ years who had undergone a previous abdominal surgery, and patients likely to experience a difficult insertion due to a colon adhesion found during a previous colonoscopy [22].

\section{Data collection and outcome parameters}

We used an electronic endoscopy reporting system (T-File System; STS Medic, Japan), which was integrated into the clinic's patient record systems, and extracted the necessary information from the endoscopy reporting system into a Microsoft Excel file with automatic data transfer [36].

Patient age, sex [37], indication for colonoscopy (i. e., evaluation of symptoms, screening, polyp surveillance) [15,38], the type of colonoscope (290 series or 260 series) [21], and bowel preparation grade (A, B, or C) [19] were collected as the baseline patient characteristics [23,30,39].

As outcome parameters, we collected the number of detected polyps, the polyp detection rates, and the colonoscopy procedures before and after the feedback. The numbers of detected polyps involved MAP, MAP by location, MAP by morphology, MAP by size, MAP +, mean number of advanced adenomas, and mean number of CSSPs. The polyp detection rates included ADRs, advanced ADRs, low-risk ADRs, high-risk ADRs, and CSSP detection rates (CSSDRs). The colonoscopy procedures included cecal insertion time and withdrawal time. The withdrawal time included the time required for the polypectomy.

\section{Statistical analysis}

To reduce the effects of selection bias and potential confounding factors in this study, we performed rigorous adjustments for significant differences in all baseline patient characteristics using propensity-score matching. Patients who underwent colonoscopy before the feedback were identified and propensityscore matched with those who underwent colonoscopy after the feedback. Matching was performed with a 1:1 matching protocol using nearest-neighbor matching without replacement and with a caliper width of 0.25 of the pooled standard deviation of logit of the propensity score.

Following the propensity-score matching, we analyzed the differences of the numbers of detected polyps, the polyp detection rates, and the colonoscopy procedures between two groups. We assessed $P$ values using the Wald test with logistic regression. Statistical significance was considered a two-sided $P<0.05$.

All statistical analyses were performed using statistical software Ekuseru-Toukei 2015 (Social Survey Research Information Co., Ltd., Tokyo, Japan).

\section{Results}

\section{Baseline characteristics}

Eleven endoscopists were eligible for this study. As shown in - Fig. 1, A total of 874 patients before the feedback and 1,272 patients after the feedback were identified as individuals who met our inclusion criteria. Prior to propensity-score matching, there were differences between the two groups before and 


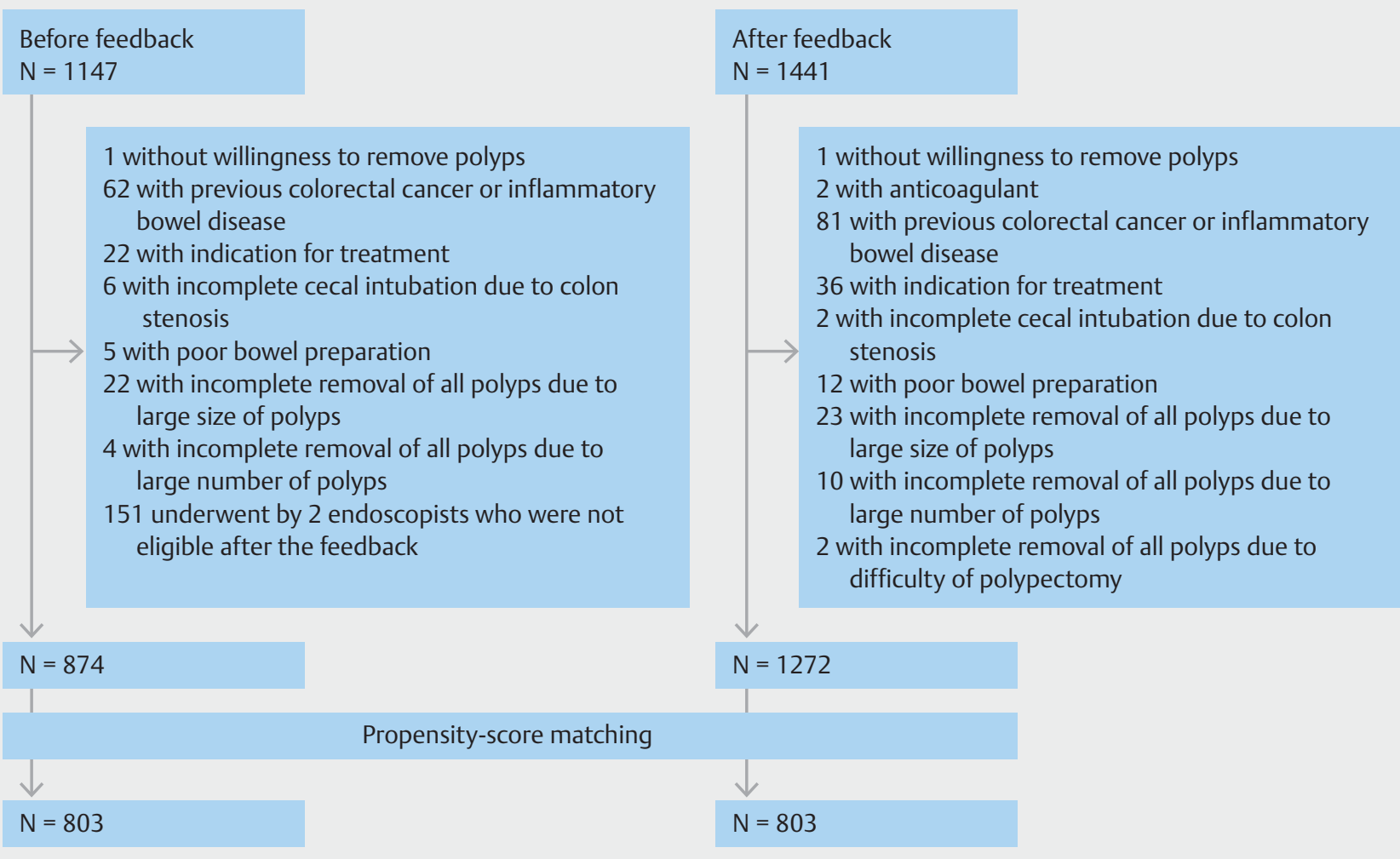

- Fig. 1 Flowchart of patient enrollment.

- Table 1 Baseline characteristics before and after propensity-score matching.

\begin{tabular}{|c|c|c|c|c|}
\hline & \multicolumn{2}{|l|}{ Before matching } & \multicolumn{2}{|l|}{ After matching } \\
\hline & Before feedback & After feedback & Before feedback & After feedback \\
\hline No. & 874 & 1272 & 803 & 803 \\
\hline Age, y (standard deviation) & $52.8(12.8)$ & $54.9(12.3)$ & $53.1(12.6)$ & $53.5(12.6)$ \\
\hline Male sex, \% & 46.2 & 48.2 & 46.8 & 47.3 \\
\hline Indication, $\mathrm{A} / \mathrm{B} / \mathrm{C}^{1}$ & $214 / 410 / 250$ & $242 / 570 / 460$ & $180 / 389 / 234$ & $192 / 358 / 253$ \\
\hline Colonoscope, $290 / 260^{2}$ & $756 / 118$ & $1216 / 56$ & $749 / 54$ & $747 / 56$ \\
\hline Bowel preparation, $\mathrm{A}$ or $\mathrm{B} / \mathrm{C}^{3}$ & $772 / 102$ & $1135 / 137$ & $709 / 94$ & $705 / 98$ \\
\hline \multicolumn{5}{|c|}{$\begin{array}{l}1 \text { Indication: A, evaluation of symptoms; B, screening; C, surveillance. } \\
{ }^{2} \text { Colonoscope type: } 290 \text {, Olympus CF-HQ290Z, CF-HQ290, or PCF-H290Z; 260, Olympus PCF-PQ260 or CF-H260. } \\
{ }^{3} \text { Bowel preparation: A, all colon segments empty an clean or minor amount of fluid in the gut, but easily removed by suction; B, at least one colon segment with } \\
\text { residual amounts of brown liquid or semi-solid stool that could be easily removed or displaced; C, at least one colon segment with only partially removable stool } \\
\text { preventing complete visualization of mucosa. }\end{array}$} \\
\hline
\end{tabular}

after the feedback in the baseline characteristics of age, indication for colonoscopy, the colonoscope used, and bowel preparation grade ( $>$ Table 1 ). Using propensity-score matching, 803 patients before the feedback and 803 patients after the feedback were matched. After matching, the mean age before and after the feedback were 53.1 and 53.5 years, respectively, and there were $46.8 \%$ and $47.3 \%$ males, respectively.

\section{Outcomes before and after feedback}

Polyp detection and the colonoscopy procedure before and after feedback are shown in $>$ Table 2. MAP after the feedback was larger than that before the feedback (0.92 and 0.69, respectively), as well as MAP+ (1.96 and 1.69, respectively). MAPs in both locations, a MAP of type 0 -II morphology, and MAPs of all size categories significantly increased after the feedback. The number of CSSPs after the feedback was larger 
- Table 2 Polyp detection and colonoscopy procedure before and after feedback.

\begin{tabular}{|c|c|c|c|c|}
\hline & $\begin{array}{l}\text { Before feedback } \\
n=803\end{array}$ & $\begin{array}{l}\text { After feedback } \\
n=803\end{array}$ & Difference & $P$ value \\
\hline \multicolumn{5}{|l|}{ No. of detected polyps (SD) } \\
\hline - Adenomas=MAP & $0.69(1.06)$ & $0.92(1.25)$ & 0.23 & $<0.001$ \\
\hline \multicolumn{5}{|l|}{ By location } \\
\hline - Proximal colon & $0.48(0.96)$ & $0.65(1.03)$ & 0.17 & $<0.001$ \\
\hline - Distal colon and rectum & $0.22(0.53)$ & $0.28(0.57)$ & 0.06 & 0.027 \\
\hline \multicolumn{5}{|l|}{ By morphology } \\
\hline - Type 0-ı & $0.07(0.29)$ & $0.08(0.29)$ & 0.00 & 0.932 \\
\hline - Type 0-II & $0.62(0.99)$ & $0.85(1.16)$ & 0.23 & $<0.001$ \\
\hline \multicolumn{5}{|l|}{ By size } \\
\hline . $\leq 5 \mathrm{~mm}$ & $0.62(1.00)$ & $0.75(1.11)$ & 0.13 & 0.013 \\
\hline - $6-9 \mathrm{~mm}$ & $0.06(0.24)$ & $0.13(0.36)$ & 0.07 & $<0.001$ \\
\hline . $\geq 10 \mathrm{~mm}$ & $0.01(0.12)$ & $0.05(0.22)$ & 0.03 & $<0.001$ \\
\hline Adenomas per positive procedure $=\mathrm{MAP}+$ & $1.69(1.04)$ & $1.96(1.28)$ & 0.27 & 0.004 \\
\hline Advanced adenomas & $0.02(0.13)$ & $0.05(0.24)$ & 0.03 & $<0.001$ \\
\hline CSSPs & $0.13(0.41)$ & $0.17(0.44)$ & 0.05 & 0.032 \\
\hline \multicolumn{5}{|l|}{ Polyp detection rate, \% } \\
\hline - ADR & 40.8 & 50.8 & 10.0 & $<0.001$ \\
\hline - Advanced ADR & 1.7 & 4.7 & 3.0 & 0.001 \\
\hline - Low-risk ADR & 33.3 & 36.7 & 3.5 & 0.143 \\
\hline - High-risk ADR & 7.6 & 14.1 & 6.5 & $<0.001$ \\
\hline - CSSP detection rate $=$ CSSDR & 10.0 & 14.9 & 5.0 & 0.003 \\
\hline \multicolumn{5}{|l|}{ Colonoscopy procedure, $\min$ (SD) } \\
\hline - Insertion time & $4.70(2.57)$ & $4.47(2.79)$ & -0.23 & 0.081 \\
\hline - Withdrawal time & $13.32(3.98)$ & $12.92(3.44)$ & -0.40 & 0.032 \\
\hline
\end{tabular}

than that before the feedback ( 0.17 and 0.13 , respectively). ADR after the feedback was significantly higher than that before the feedback ( $50.8 \%$ and $40.8 \%$, respectively). Fig. 2 shows the change in adenoma detection rate for each endoscopist following feedback. High-risk ADR significantly improved from $7.6 \%$ to $14.1 \%$ after the feedback. CSSDR was also improved from $10.0 \%$ to $14.9 \%$.

The withdrawal time after the feedback was shorter than that before the feedback (12.92 and $13.32 \mathrm{~min}$, respectively), while the insertion time after the feedback was similar to that before the feedback (4.47 and $4.70 \mathrm{~min}$, respectively).

\section{Discussion}

This study showed that a simple feedback of colonoscopy performance improved not only the ADR but also the MAP, MAP+, and CSSDR. Furthermore, this is the first propensity-score matching study that demonstrated improvement of colonoscopy performance before and after the feedback.

Our feedback method had some unique characteristics. First, feedback of colonoscopy performance included not only the ADR but also the MAP and MAP+. Second, we evaluated the CSSDR as well as ADR. Third, the ranking of colonoscopy performance was announced during our meeting.

As the first point, the feedback included not only the ADR but also MAP and MAP+. Focus on ADR could lead an "one and done" phenomenon [14]. Feedback of the MAP and $\mathrm{MAP}+$ may have suppressed the "one and done" phenomenon. 


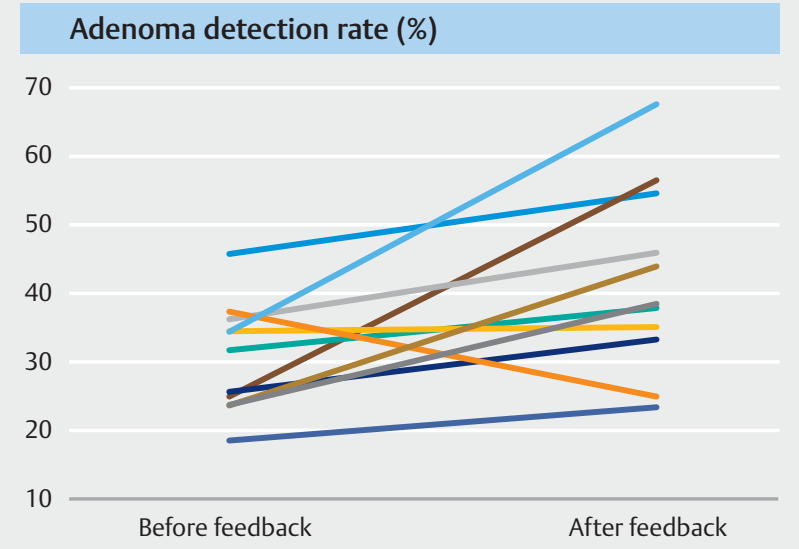

- Fig. 2 Change in adenoma detection rate per endoscopist following feedback.

Zhao et al. [19] reported that a MAP+ was an important indicator of colonoscopy quality, because MAP + was independently associated with both adenoma miss rate and advanced adenoma miss rate, whereas ADR or MAP was not. They also described that MAP $+>1.8$ was more effective in monitoring the adenoma miss rate. Our MAP + value achieved the criteria.

As the second point, we evaluated the CSSDR as well as the ADR and showed that just feedback without training made CSSDR rise. Bleijenberg et al. [16] reported educational interventions improved proximal SP detection rate. However, our study indicated that just feedback might be enough.

As the third point, the announcement of ranking may have aroused a spirit of competition for endoscopists. This may have contributed to the astonishing ADR of $50.8 \%$ after the feedback. We improved ourselves through friendly rivalry for the purpose of preventing CRC. This study was examined in a facility specialized in endoscopy, which already had a high average ADR of $40 \%$ before the feedback. Gurudu et al. [11] defined ADR of $35 \%$ or more as high detectors, and Hilsden et al. [40] defined ADR of $40 \%$ or more as aspirational detectors. The clinic exerted to improve ADR including the bundle (withdrawal time of 6 or more minutes, use of scopolamine butylbromide, use of supine position for examination of the transverse colon, and rectal retroflexion). Thus, this study would serve as an indicator for medical institutions especially with developing ADR.

This study examined the MAP by location, morphology, and size. All MAPs by location and size increased after the feedback; however, only a MAP of type 0 -II in the morphology improved after the feedback. Our study indicates that detection of more adenomas of type 0-II may contribute to improved ADR. In addition, Zimmermann-Fraedrich et al. [37] and Zhao et al. [19], similar to our previous study [22], stated that right-side, flat, and diminutive adenomas were often missed in colonoscopy. Similar results were obtained in this study, which is a comparison of the same endoscopist before and after the feedback. We consider these findings to be useful information for improving the ADR in clinical practice.
The present study has several limitations. This study was conducted at a single institution. However, the institution was a facility specialized in endoscopy, exerted to improve ADR including the bundle, and had average ADR before feedback as high as $40 \%$. This study will be helpful for medical institutions with developing ADR; however, validation at multiple institutions is required. This study excluded patients with large-size or a number of the lesions because colonoscopy was conducted in an outpatient institution. Therefore, data on advanced adenomas and high-risk adenoma were surrogate. In this study, we compared data before and after feedback in a short period. The long-term effect of the feedback is controversial $[12,16]$. Additionally, we did not clarify whether the simple feedback given only once or just the fact of being continuously observed after the feedback improved the studied outcomes. Further investigations, using investigational study designs such as randomized controlled trials are needed in the future.

\section{Conclusions}

In conclusion, feedback including ADR, MAP, MAP+, and CSSDR could improve on those quality indicators. Feedback could be vital to self-motivated quality improvement in endoscopic practice. Detection of more flat adenomas may contribute to increased ADR. More studies are needed to determine optimal feedback for high colonoscopy performance in clinical practice.

\section{Competing interests}

The authors declare that they have no conflict of interest.

\section{References}

[1] Hoff G, Botteri E, Hoie O et al. Polyp detection rates as quality indicator in clinical versus screening colonoscopy. Endosc Int Open 2019; 7: E195-E202

[2] Robertson DJ, Lieberman DA, Winawer S] et al. Colorectal cancers soon after colonoscopy: a pooled multicohort analysis. Gut 2014; 63: 949-956

[3] Kaminski MF, Regula J, Kraszewska E et al. Quality indicators for colonoscopy and the risk of interval cancer. N Engl J Med 2010; 362: 1795-1803

[4] Corley DA, Jensen CD, Marks AR et al. Adenoma Detection rate and risk of colorectal cancer and death. N Engl J Med 2014; 370: 12981306

[5] Brenner H, Chang-Claude J, Seiler CM et al. Protection from colorectal cancer after colonoscopy: a population-based, case-control study. Ann Intern Med 2011; 154: 22-30

[6] Arain MA, Sawhney M, Sheikh S et al. CIMP status of interval colon cancers: another piece to the puzzle. Am J Gastroenterol 2010; 105 : 1189-1195

[7] Nalankilli K, Huynh XT, Lade S et al. Increasing rates of SSA/P detection in a large open-access Australian colonoscopy cohort. Endosc Int Open 2019; 7: E310-E316

[8] East JE, Atkin WS, Bateman AC et al. British Society of Gastroenterology position statement on serrated polyps in the colon and rectum. Gut 2017; 66: 1181-1196 
[9] Bettington M, Walker N, Rosty C et al. Clinicopathological and molecular features of sessile serrated adenomas with dysplasia or carcinoma. Gut 2017; 66: 97-106

[10] Coe SG, Crook JE, DiehI NN et al. An endoscopic quality improvement program improves detection of colorectal adenomas. Am J Gastroenterol 2013; 108: 219-226

[11] Gurudu SR, Boroff ES, Crowell MD et al. Impact of feedback on adenoma detection rates: Outcomes of quality improvement program. J Gastroenterol Hepatol 2018; 33: 645-649

[12] Neilson LJ, East JE, Rajasekhar PT et al. Sustained colonoscopy quality improvement using a simple intervention bundle. Endoscopy 2020; 52: $285-292$

[13] Mahadev S, Jin Z, Lebwohl B et al. Trainee colonoscopy quality is influenced by the independent and unobserved performance characteristics of supervising physicians. Endosc Int Open 2019; 7: E74-E82

[14] Ussui V, Coe S, Rizk C et al. Stability of increased adenoma detection at colonoscopy. Follow-up of an endoscopic quality improvement program-EQUIP-II. Am J Gastroenterol 2015; 110: 489-496

[15] Li D, Woolfrey J, Jiang SF et al. Diagnosis and predictors of sessile serrated adenoma after educational training in a large, communitybased, integrated healthcare setting. Gastrointest Endosc 2018; 87: 755-765.e751

[16] Bleijenberg AGC, van Leerdam ME, Bargeman M et al. Substantial and sustained improvement of serrated polyp detection after a simple educational intervention: results from a prospective controlled trial. Gut 2020; 69: 2150-2158

[17] Lam AY, Li Y, Gregory DL et al. Association between improved adenoma detection rate and interval colorectal cancer rates after a quality improvement program. Gastrointest Endosc 2020; 92: 355-364

[18] Fayad NF, Kahi C]. Quality measures for colonoscopy: a critical evaluation. Clin Gastroenterol Hepatol 2014; 12: 1973-1980

[19] Zhao S, Wang S, Pan P et al. Magnitude, Risk factors, and factors associated with adenoma miss rate of tandem colonoscopy: a systematic review and meta-analysis. Gastroenterology 2019; 156: 1661-1674.e1611

[20] Fedewa SA, Anderson JC, Robinson CM et al. Prevalence of 'one and done' in adenoma detection rates: results from the New Hampshire Colonoscopy Registry. Endosc Int Open 2019; 7: E1344-E1354

[21] Toyoshima O, Yoshida S, Nishizawa T et al. CF290 for pancolonic chromoendoscopy improved sessile serrated polyp detection and procedure time: a propensity score-matching study. Endosc Int Open 2019; 7: E987-E993

[22] Toyoshima O, Nishizawa T, Yoshida S et al. Expert endoscopists with high adenoma detection rates frequently detect diminutive adenomas in proximal colon. Endosc Int Open 2020; 8: E775-E782

[23] Anderson JC, Butterly LF, Weiss JE et al. Providing data for serrated polyp detection rate benchmarks: an analysis of the New Hampshire Colonoscopy Registry. Gastrointest Endosc 2017; 85: 1188-1194

[24] Klair JS, Ashat M, Johnson D et al. Serrated polyp detection rate and advanced adenoma detection rate from a US multicenter cohort. Endoscopy 2020; 52: 61-67
[25] Rex DK, Ahnen DJ, Baron JA et al. Serrated Lesions of the Colorectum: Review and Recommendations From an Expert Panel. Am J Gastroenterol 2012; 107: 1315-1330

[26] Kashida H. Endoscopic diagnosis of sessile serrated polyp: A systematic review. Dig Endosc 2019; 31: 16-23

[27] Nagtegaal I, Arends MJ, Odeze RD et al. Tumours of the colon and rectum. In: The WHO Classification of Tumours Editorial Board (eds). WHO Classification of Tumours Digestive System Tumours. Lyon (France): International Agency for Research on Cancer (IARC); 2019

[28] Park SK, Kim HS, Yang HJ et al. Coexistent adenoma and serrated polyps on index colonoscopy and the risk of metachronous advanced colorectal neoplasia. Endosc Int Open 2019; 7: E1748-E1754

[29] Lieberman DA, Rex DK, Winawer S] et al. Guidelines for colonoscopy surveillance after screening and polypectomy: a consensus update by the US Multi-Society Task Force on Colorectal Cancer. Gastroenterology 2012; 143: 844-857

[30] Lee T], Rees C], Blanks RG et al. Colonoscopic factors associated with adenoma detection in a national colorectal cancer screening program. Endoscopy 2014; 46: 203-211

[31] Nishizawa T, Suzuki H, Arita M et al. Pethidine dose and female sex as risk factors for nausea after esophagogastroduodenoscopy. J Clin Biochem Nutri 2018; 63: 230-232

[32] Nishizawa T, Sakitani K, Suzuki H et al. Adverse events associated with bidirectional endoscopy with midazolam and pethidine. J Clin Biochem Nutr 2019; 66: 78-81

[33] Toyoshima O, Hata K, Yoshida S et al. New-generation chromoendoscopy may increase confidence in the DISCARD2 study. Gut 2018; 67: 1742-1743

[34] Rajasekhar PT, Rees C], Bramble MG et al. A multicenter pragmatic study of an evidence-based intervention to improve adenoma detection: the Quality Improvement in Colonoscopy (QIC) study. Endoscopy 2015; 10: 217-224

[35] Ell C, Fischbach W, Bronisch HJ et al. Randomized trial of low-volume PEG solution versus standard PEG + electrolytes for bowel cleansing before colonoscopy. Am J Gastroenterol 2008; 103: 883-893

[36] Bretthauer M, Aabakken L, Dekker E et al. Requirements and standards facilitating quality improvement for reporting systems in gastrointestinal endoscopy: European Society of Gastrointestinal Endoscopy (ESGE) Position Statement. Endoscopy 2016; 48: 291-294

[37] Zimmermann-Fraedrich K, Sehner S, Rex DK et al. Right-sided location not associated with missed colorectal adenomas in an individual-level reanalysis of tandem colonoscopy studies. Gastroenterology 2019; 157: 660-671.e662

[38] Gimeno García AZ, González Y, Quintero E et al. Clinical validation of the European Panel on the Appropriateness of Gastrointestinal Endoscopy (EPAGE) II criteria in an open-access unit: a prospective study. Endoscopy 2012; 44: 32-37

[39] van Doorn SC, Klanderman RB, Hazewinkel Y et al. Adenoma detection rate varies greatly during colonoscopy training. Gastrointest Endosc 2015; 82: 122-129

[40] Hilsden RJ, Rose SM, Dube C et al. Defining and applying locally relevant benchmarks for the adenoma detection rate. Am J Gastroenterol 2019; 114: 1315-1321 